\title{
DESIGUALDADES CONECTADAS: ANÁLISIS ESTRUCTURAL DEL PLAN CONECTAR IGUALDAD
}

\author{
Victoria Matozo \\ Instituto de Investigaciones Gino Germani Universidad de Buenos Aires (UBA) \\ Consejo Nacional de Investigaciones Científicas y Técnicas (CONICET) \\ victoria.matozo@gmail.com
}

Para citar este artículo:

Matozo, V. (2019). Desigualdades conectadas: análisis estructural del Plan Conectar Igualdad. Commons. Revista de Comunicación y Ciudadanía Digital, 8(1), 76-119

http://dx.doi.org/10.25267/COMMON.2019.v8.i1.03

Fecha de recepción: 16/07/2018. Fecha de aceptación: 29/12/2018

\section{Resumen}

Este trabajo realiza una tipología de los alumnos beneficiarios del Plan Conectar Igualdad (PCI), la política pública argentina de inclusión digital educativa, a partir de un Análisis de Correspondencias Múltiples (ACM).

Este análisis cuantitativo se centra en los conceptos de representaciones sociales, la brecha digital y el acceso a TIC (Tecnologías de la Información y la Comunicación), y el nivel socioeconómico de los estudiantes de nivel secundario beneficiarios del PCI. Los resultados del mismo permiten analizar concordancias y diferencias entre los grupos creados e identificar la influencia de la posición en la estructura social de estos individuos en la formación de los tipos.
CONNECTED INEQUALITIES: STRUCTURAL ANALYSIS OF PLAN CONECTAR IGUALDAD 


\section{Introducción}

Este artículo analiza la implementación del programa de inclusión digital Plan Conectar Igualdad (PCI) en los estudiantes de Escuelas Medias de gestión pública de la República Argentina. El análisis cuantitativo realizado pretende comenzar un camino de indagación y reflexión sobre el impacto del PCI en nuestros estudiantes.

El Plan Conectar Igualdad es el programa argentino que siguiendo el modelo educativo digital 1:1 (un dispositivo por alumno) entregaba una netbook a cada docente y estudiante de escuelas secundarias, de educación especial y de institutos de formación docente de gestión estatal, con el fin de reducir las desigualdades en el acceso a la tecnología y fomentar la alfabetización tecnológica ${ }^{1}$. El objetivo de inclusión digital y el acceso a la tecnología parecía ser el eje principal de esta política, dado que comenzó siendo dirigida y administrada por un organismo de Seguridad Social (Administración Nacional de la Seguridad Social, ANSES). Su creación se relacionó con el derecho a la información, y las netbooks que el plan entregó se dieron en forma de comodato mientras el estudiante concurriera a la escuela, cuando este se graduara la computadora se desbloqueaba indefinidamente y pasaba a ser propiedad privada del individuo. Sin embargo, numerosos cambios redefinieron a esta política hasta su finalización en el 2018. Dentro de este panorama, este trabajo se ubica en un período anterior al presente, con el fin de acercarnos más a la evaluación del impacto del PCI en los estudiantes hasta el 2015, y a describir diferencias y similitudes en las experiencias y representaciones sociales de los alumnos en un período en el que el Plan Conectar Igualdad estaba en funcionamiento en Argentina.

Durante el periodo de funcionamiento (2010-2018) Conectar Igualdad entregó más de 6 millones de netbooks y llegó a ser la política pública de modelo 1:1 de mayor cantidad de dispositivos distribuidos del mundo.

1. Relevado en www.conectarigualdad.gob.ar el 09/08/2016. 
El objetivo del presente trabajo es realizar una tipología de los perfiles de estudiantes beneficiarios del Plan Conectar Igualdad (PCI) y usuarios de las netbooks del programa durante el año 2015 utilizando una metodología cuantitativa, en relación a tres ejes principales: el nivel socioeconómico del alumno, el acceso a TIC, y las representaciones sociales sobre diferentes aspectos del PCI. El interés por rastrear las relaciones entre estos tres ejes se basa en intentar superar perspectivas parciales que solo analizan la implementación del PCI unidimensionalmente.

Finalmente el análisis cuantitativo que se plantea es el Análisis de Correspondencias Múltiples, explicando los procedimientos y resultados del mismo a fin de generar un mayor entendimiento sobre la problemática a estudiar en este artículo.

\section{Antecedentes de inclusión digital y desarrollos teóricos en torno a la relación entre usuario y tecnología}

La revolución tecnológica planteada por Manuel Castells (1997) es el contexto en el que este trabajo se inscribe: los cambios sociales propiciados por la inclusión de TIC (Tecnologías de la Información y la Comunicación) en la cotidianidad, han dado origen a una nueva etapa denominada Sociedad de la Información o Capitalismo Cognitivo o Informacional (Zukerfeld, 2010). A partir de esta revolución, se hizo evidente la existencia de una "brecha digital", en dos sentidos: una brecha internacional que compara diferentes países en cuanto a la difusión de la tecnología, y una brecha doméstica que presenta desigualdades en el acceso a la tecnología al interior de los países (CEPAL, 2003: 23-24) en términos de acceso y uso de sus habitantes.

El concepto de brecha digital surgió durante el gobierno de Bill Clinton a finales de los años 90 en los documentos Falling throught the net que remitían a la fractura social entre "conectados" y "no conectados". La brecha se definió en sus orígenes 
como una brecha de acceso a la tecnología (Camacho, 2005), quien tiene y quien no. Este paradigma simplista ha sido fuertemente criticado desde sus orígenes, dando lugar a redefiniciones y estudios que se centraron en los usos de las tecnologías digitales (Di Maggio et al., 2001) o en el mero acceso o disponibilidad de las mismas.

Dentro de las críticas hacia esta postura, considero central el aporte de Neil Selwyn (2004) cuyo planteamiento superador de esta dicotomía redefine la brecha digital como un concepto continuo, de diferentes niveles jerárquicos de engagement con las tecnologías digitales (TD). Si bien resulta confusa la traducción del nivel de engagement, podría decirse que se trata del grado en el que un sujeto está enganchado o prendido con la tecnología, cuán implicado está con la misma. Este es el punto sobre el cual se construye el concepto de brecha digital de manera no binaria, y será el concepto de apropiación el que permita acceder a los niveles de engagement de manera empírica. Más allá del acceso y el uso, la apropiación se corresponde con un nivel superior del estudio de la brecha digital tal como lo convive Selwyn (2004). Este concepto, utilizado por primera vez por John Thompson (1990: 463), se refiere a un proceso de "hacer propio" algo nuevo. En este sentido:

\footnotetext{
El proceso de 'hacer propiedad de uno' debe entenderse en relación con los individuos particulares que, en el curso de sus vidas diarias, reciben los mensajes mediados, hablan acerca de ellos con los demás, y que, a través de un proceso continuo de elaboración discursiva, los integran a sus vidas.
}

Si bien es el concepto de apropiación el que considero central a indagar en futuras investigaciones a través de metodologías cualitativas, la brecha digital, sus críticas y redefiniciones son centrales para entender la trama de desigualdad en la cual están insertos los sujetos en relación a la tecnología. Es en este sentido, investigaciones feministas han retomado el concepto de brecha para hacer evidente la existencia 
de una segunda brecha digital: la brecha de género. Los roles de género en torno a la tecnología están definidos desde la niñez. Como explica David Morley, el objetivo no es crear una descripción esencialista sobre los usos o apropiaciones digitales/mediáticas de hombres y mujeres, sino que "la cuestión esencial es la incorporación de las tecnologías en esos modelos definidos culturalmente" (Morley, 1996: 334). Martínez-Reina y Vélez Cea (2010) explican la llegada de la computadora a los jóvenes, primero en forma de juego en la infancia diferenciadas por sexo. Estos autores advierten que las tecnologías orientadas a los niños ofrecen gran variedad de juegos y consolas con personajes masculinos como protagonistas, mientras que las niñas por su parte no logran la identificación con los personajes femeninos secundarios o degradados de estos juegos, con lo cual, ellas se alejan de dichos consumos y eligen otro tipo de actividades lúdicas. Esta situación de oferta de juegos tecnológicos influye en que sean los varones los que a largo plazo estén más familiarizados con la tecnología (Martínez-Reina \& Vélez Cea, 2010: 12).

En Argentina, la Fundación Sadosky ha realizado en 2013 un informe titulado " ¿Y las mujeres dónde están?", en relación a la participación femenina en el campo informático. En este informe, la brecha de género fue evidenciada a partir de las representaciones sociales que los individuos (todos trabajadores del campo informático) tienen sobre hombres y mujeres en relación a la tecnología.

Cada mujer entrevistada se percibe a sí misma, en promedio, como mucho más ajena a la informática que como se percibe en promedio a sí mismo cada varón. Tanto los varones como las mujeres, opinando no sobre sí mismos, sino sobre los géneros en general, sitúan a las mujeres lejos de la informática. (Fundación Sadosky, 2013: 84)

El reconocimiento del campo informático como un área masculinizada será retomado en este estudio al incluir la variable género dentro del análisis. 
Por otro lado y desde una perspectiva de derechos, el acceso a la tecnología se corresponde con la perspectiva de derechos sociales de las Naciones Unidas que surge a partir de los derechos humanos: es así como las políticas públicas deberían tender a incluir los derechos humanos en los objetivos de reducción de pobreza y desigualdades. En esta línea, la Asamblea General de las Naciones Unidas declaró el 29 de junio de 2012 el acceso a internet como un derecho humano por su condición de herramienta que favorece el crecimiento y "promueve el progreso de la sociedad en su conjunto"2. La ONU ha enmarcado este derecho dentro del artículo 19 de la Declaración Universal de los Derechos Humanos sobre derecho a la información, que establece el derecho a "investigar y recibir informaciones y opiniones, y el de difundirlas, sin limitaciones de fronteras, por cualquier medio de expresión" (Declaración Derechos Humanos, 1948). Otros organismos definieron de forma similar el acceso a internet como por ejemplo la Organización de Estados Americanos (OEA), que el 1 de junio de 2011 realizó una Declaración Conjunta sobre Libertad de Expresión e internet estableciendo que los Estados "tienen la obligación de promover el acceso universal a internet para garantizar el disfrute efectivo del derecho a la libertad de expresión" (Comunicado de prensa R50/11). Las definiciones de estos organismos internacionales se relacionan con el objetivo de atacar desigualdades en materia de tecnología reduciendo la brecha digital que se genera en la Sociedad de la Información, meta promovida por Unicef, ONU y el Banco Mundial. Estos organismos también enfatizan la importancia de la inclusión educativa de TIC, siendo la educación un ámbito prioritario de modernización tecnológica, por lo que en muchos países las políticas sociales de acceso a tecnología se dieron de la mano de políticas educativas. Como primera hipótesis podría suponerse que dado que el acceso a internet se da a través de las TIC, los Estados solo pueden asegurar este derecho proveyendo los dispositivos tecnológicos que permitan conectarse a internet. En cuanto a los servicios universales que nombra (pero no define concretamente) la ley de Argentina Digital plantea que el acceso a "servicios TIC" deberán prestarse a un precio justo y razonable, derivando implícitamente la provisión de los servicios al mercado, pero abriendo una puerta para las políticas públicas en relación a la tecnología.

2. Declaración del Relator Especial de la ONU, Frank La Rue, en la sesión 17 del Consejo de Derechos Humanos de la ONU (16 de mayo del 2011) dentro del informe Report of the Special Rapporteur on the promotion and protection of the right to freedom of opinion and expression. Consultado el 21/03/2018 en http://www2.ohchr.org/english/bodies/hrcouncil/docs/17session/A.HRC.17.27_en.pdf 
Es así como a nivel global, los gobiernos comenzaron a incluir el tema en sus agendas con vistas a generar políticas sociales que mejoren el uso y el acceso de TIC en la sociedad. En este proceso ha sido el campo educativo el protagonista de estas políticas. En primer lugar, esto se debió a que las políticas de inclusión digital se focalizaron en los jóvenes desde una perspectiva educativa. Y en segundo lugar se privilegió a la escuela por ser un ámbito donde la difusión de tecnología no era una temática ajena al funcionamiento de dicha institución. Los primeros intentos de incluir tecnología en las instituciones educativas se dieron en la década de 1990 con los laboratorios de computación, salones dotados de computadoras en donde el uso de tecnología se restringía a dicho espacio físico, a un horario definido y a una signatura (computación o informática) definida. Si bien hubo variantes en la implementación de dichos laboratorios, todos coincidían en acotar el conocimiento digital y recortarlo a un lugar y momento definido.

El cambio de paradigma se dio con la presentación del profesor del MIT (Massachusetts Institute of Technology) Nicholas Negroponte en Davos, Suiza, del año 2006. Durante el encuentro, Negroponte presentó la iniciativa One Laptop per Child (OLPC) (Una Laptop por Niño), programa que distribuye notebooks educativas a estudiantes con el fin de mejorar la educación a través de la tecnología. Hoy en día diferentes países han seguido esta iniciativa, implementando el mismo programa en su territorio o creando programas similares bajo el modelo 1:1 de una computadora por alumno. Este modelo 1:1 propuso un cambio en la concepción de los conocimientos en computación, vistos ahora como un conocimiento transversal (Artopoulos y Kozak, 2001: 5) a todas las asignaturas, y proponiendo una utilización ubicua (en cualquier momento y lugar) de la tecnología, borrando las fronteras espaciales y temporales que proponían los laboratorios de computación de los 90. El Plan Ceibal de Uruguay fue pionero en Latinoamérica en implementar una política pública de inclusión educativa de TIC bajo el modelo 1:1. Este programa implementado en el 2006, comenzó como un plan piloto en la localidad de Villa Cardal (mayo 2007) que fue expandido a nivel nacional completando los niveles primario y secundario con un modelo 1:1 (Vacchieri, 2013: 63). Se convirtió en un referente mundial por la cobertura del plan (que llegó a alcanzar el $100 \%$ de la matrícula escolar uruguaya) y por ser un proyecto pionero que "ha marcado cierta trayectoria tecnológica en la región" 
(Dughera 2015: 102). Otros Estados pidieron asistencia técnica al Plan Ceibal tal como ocurrió con el Proyecto Piloto "Mi Compu" en la ciudad de Cuenca, Ecuador (2011). Argentina si bien en un principio analizó las propuestas de One Laptop per Child y Ceibal, decidió realizar una apuesta propia al crear el Plan Conectar Igualdad (PCI).

Diferentes organismos internacionales como el Fondo de las Naciones Unidas para la Infancia (UNICEF) y la UNESCO, han realizado informes sobre la inclusión de TIC en las escuelas latinoamericanas (Cfr. Vacchieri, 2013), en los cuales se le reconoce a Argentina el haber incorporado tecnología con gran rapidez creando un proyecto de inclusión digital ambicioso. Sin embargo, estos informes señalan que la inversión y la rápida respuesta al acceso a la tecnología no se ha visto traducida en mejores resultados educativos, proponiendo un enfoque de "brecha digital" orientada más al uso que al acceso (Tedesco, 2005; Levis, Diéguez \& Rey, 2011; Buckingham, 2008). Es así como las investigaciones realizadas suelen tratarse de comparaciones entre escuelas que incluyen mejor o peor las TIC, comparaciones entre diferentes implementaciones de los modelo 1:1 (Cfr. Area Moreira y otros, 2014), experiencias docentes y estudiantiles (Cfr. Ross, 2014; Lago Martínez, 2012, entre otros), el rol "prosumidor" de los alumnos (Frau Meigs, 2011), experiencias áulicas o casos de éxito, pero no de analizar el panorama completo de qué ocurre con los alumnos cuando entran en el PCI y disponen de una netbook personal los 365 días del año.

El impacto del PCI en Argentina ha sido un tema poco estudiado en general, por ello resulta de nuestro interés la temática y el foco puesto en los alumnos como protagonistas, pero no como productores o desde una mirada que exaltelas "buenas prácticas" escolares con TIC, sino el alumno a través de sus representaciones sociales (en la base de datos denominadas "percepciones") sobre el PCI y la calidad de su funcionamiento. Asimismo, las diferentes formas de apropiación de la tecnología, como las representaciones sociales de los actores sobre la misma y sobre el uso que estos hacen de las TIC, no ha sido hasta el momento un tema de gran interés para las investigaciones sobre tecnología que incluso, muchas veces, suponen cierta homogeneidad dentro de un mismo grupo social, un curso o una escuela, invisibilizando de esta forma las diferencias interclase. 
En este sentido, Sebastián Benítez Larghi (2017) retomando a Helsper (2017) y Van Deursen, Helsper, Eynon y van Dijk (2017), señala que:

\begin{abstract}
Usuarios con el mismo bagaje sociocultural y económico y las mismas habilidades digitales obtienen provechos y consiguen recursos diferentes (...) desde una perspectiva latinoamericana de los estudios culturales, diversos trabajos e investigaciones (Cabrera Paz, 2001; Winocur, 2009; Remondino, 2012; Benítez Larghi et. al. 2015) han puesto el foco en las representaciones sociales de las TIC para comprender los sentidos en que los usos y prácticas digitales constituyen modos diferenciales de apropiación. (Benítez Larghi, 2017:10)
\end{abstract}

Serán estas representaciones sociales, como forma de pensamiento social que incluye procesos "individuales y colectivos, psicológicos y sociales" (Jodelet, 1986: $474)$ las que se plantean dentro de las variables escogidas sobre las opiniones de los estudiantes acerca de habilidades docentes, estudiantiles y la evaluación de las netbooks del Plan Conectar Igualdad. En este sentido, para Rosalía Winocur "recuperar los significados de la experiencia de los sujetos es clave para entender en qué universos simbólicos se inscriben las prácticas y representaciones sobre las TIC" (2013: 62), en este caso a través de diferentes variables disponibles en la base de datos utilizada. Estas representaciones sociales a su vez estarán influenciadas por el lugar que los individuos ocupen dentro de la estructura social.

Los estudios sobre estructura social han virado desde las primeras perspectivas funcionalistas que abordaban las clases desde las capacidades y los recursos individuales que determinan roles y recompensas sociales (Parsons, 1954), a perspectivas relacionales de estratificación multidimensional (Weber, 1964) que definen las clases sociales en un sentido weberiano, focalizando en oportunidades y posiciones sociales y concibiendo a las clases relacionalmente como un sistema de mutua dependencia (Plá, 2013). Esta es la perspectiva teórica de la presente tesis, oportunidades y posiciones se relacionan en el presente y el pasado y se proyectan hacia el futuro. Dada la "oportunidad" que el PCI podría llegar a representar en términos de inclusión digital (tener una computadora personal) las posiciones sociales de los estudiantes, su clase de origen, podrían ser determinantes para el tipo de apropiación que realizarán de la netbook. 
En cuanto al hábitus y la tecnología, existen fuertes relaciones entre la posición offline y la posición online de los individuos (Van Deursen, Helsper, Eynon \& van Dijk, 2017) pero esta relación es bidireccional por lo que la actividad online puede favorecer la posición offline del individuo. El concepto de hábitus entonces resulta insuficiente para analizar esta perspectiva. Asimismo algunas investigaciones sobre apropiaciones tecnológicas afirman que las diferencias socioeconómicas y de capital cultural no son suficientes para explicar las desigualdades en la apropiación de TIC (Lago Martínez, 2015: 286) reconociendo que las políticas educativas de inclusión de TIC como el PCI cierran la brecha digital de acceso a tecnología y acercan diversas oportunidades de uso a los estudiantes pero no equiparan los usos diversos de tecnología. Sin embargo, este trabajo se propone evidenciar que los estudiantes tienen representaciones disímiles de la inclusión digital que se habría generado con esta política pública, ya que siendo todos beneficiarios del PCI, no todos aseguran poseer una computadora.

Los procesos que están entre la clase social y las representaciones sociales, ambos conceptos centrales de este trabajo, son el consumo y la mediación de los procesos socioculturales. Desde la perspectiva de Néstor García Canclini definimos el consumo como "el conjunto de procesos socioculturales en que se realizan la apropiación y los usos de los productos" (García Canclini, 1995: 42), en este punto disintiendo con la perspectiva bourdiana de hábitus, reproducción social y consumo como distinción (Bourdieu, 1979) para añadir mayor complejidad a los usos digitales por clase social. Desde la perspectiva de García Canclini, el consumo deja de ser una práctica mecánica o exenta de racionalidad. Por el contrario, diversos procesos socioculturales dotan al consumo de significados que dan cuenta de actividad por parte del sujeto. El individuo en este caso es abordado como un sujeto activo, no como un consumidor pasivo. Y estos procesos 
de significación del consumo se dan a través de las mediaciones propuestas por Jesús Martín Barbero (1987), en la cual la escuela, el grupo de pares y la familia (como ejemplos de mediadores presentes en el análisis de este artículo) actúan como mediadores de significados en los consumos individuales, en este caso, de los estudiantes en relación a la tecnología, al Plan Conectar Igualdad y al uso de la netbook. Por ejemplo, la variable que representa a la pregunta "¿con qué relacionas el PCI?" de la encuesta, da cuenta de estos procesos: no todos los estudiantes relacionan al programa con los mismos significados, existen otras variables y procesos de mediación que influirán en las respuestas. Si bien este trabajo no pretende explicar dichos fenómenos, se plantea esta línea teórica a fin de realizar un estudio cualitativo en el futuro que pueda dar cuenta de estos procesos socioculturales.

\section{Conectar Igualdad}

En Argentina el programa que llevó adelante la propuesta de inclusión digital en el ámbito educativo fue el Plan Conectar Igualdad (PCI) del año 2010. Desde el nombre de esta política pública, que se compone de dos palabras con fuerte carga simbólica, dos apreciaciones generales pueden realizarse:

"Conectar". Esta política pública se enmarca en el derecho de acceso a tecnología que, bajo el paradigma de las Naciones Unidas entendido como derecho humano, es incluido en la ley 27.078 Argentina Digital la cual establece que las disposiciones de la presente ley tienen como finalidad "garantizar el derecho humano a las comunicaciones y a las telecomunicaciones" (Art. 2 Ley 27.078). En dicha ley el Estado "garantiza el Servicio Universal" estableciendo como finalidad del mismo "posibilitar el acceso de todos los habitantes de nuestro país, independientemente de su domicilio, ingreso o capacidades, a los Servicios de TIC prestados en condiciones de calidad y a un precio justo y razonable" (Art. 18 y 19 de la Ley 27.078). 
"Igualdad". La igualdad como objetivo está presente en el nombre de esta política pública, la cual se pretende alcanzar a partir del hecho de conectar y acceder a dispositivos TIC, en este caso la netbook. El PCI se planteó como una política de inclusión digital desde sus objetivos (presentes hasta el año 2016 en la página www.conectarigualdad.gob.ar, actualmente dada de baja) por lo que su creación da cuenta de una desigualdad preexistente en relación a la tecnología: la brecha digital. Nuevamente esta política plantea una interesante cuestión en la relación entre mercado y Estado al otorgarle un bien mueble presente en el mercado a los alumnos y permitirles que al finalizar la escuela secundaria puedan retener ese bien. Toma un elemento que era del ámbito privado hasta ese momento, como es la tecnología, para constituirlo en un problema público. Hasta ese momento, la provisión de tecnología se daba en el mercado, generando desigualdades estructurales entre los alumnos que podían acceder a TIC lo cual permite un acercamiento a la cultura más amplio que aquellos que no podrían acceder a dichos bienes.

El PCI no estuvo enmarcado en otra política pública, sino que se constituyó como la primera política masiva de inclusión digital a través de la escuela secundaria: todo docente y alumno de escuelas públicas de gestión estatal de la República Argentina sería beneficiario de un dispositivo netbook personal bajo la forma legal de comodato, pasando a ser propiedad privada del usuario tras su egreso (alumnos) o jubilación (docentes). Conectar Igualdad tuvo diferentes redefiniciones a lo largo de los años, pudiendo identificar tres momentos clave durante su existencia:

- Primera etapa, de creación. Su creación en el año 2010 por el Decreto $\mathrm{N}^{\circ}$ 459/2010 asignándole la presidencia del Comité Ejecutivo y la responsabilidad de garantizar el financiamiento a la Administración Nacional de Seguro Social (ANSES). Durante esta etapa el Ministerio de educación participaba de esta política contribuyendo a fomentar el uso educativo de las netbooks en las escuelas, pero no dirigía el PCI. 
- Segunda etapa, de paso al Ministerio de Educación. El paso de ANSES al Ministerio de Educación y Deportes formando parte de un nuevo proyecto: el PLANIED, Plan Nacional de Educación Digital. El PCI pasa a formar parte de este Plan, dirigido por Educ.ar, una división del mismo Ministerio. Este paso se formaliza mediante el Decreto 1239/2016, que define al Ministerio de Educación como el "ámbito natural" del PCI.

- Tercera etapa, fin y reconversión. El fin de Conectar Igualdad como programa en mayo de 2018. A partir del Decreto 386/2018 se crea el Plan Aprender Conectados (PAC), definido como un proyecto pedagógico de alfabetización digital. Si bien este decreto no elimina completamente al PCI lo incluye y lo redefine como el Plan que se encarga de dotar a las escuelas de equipamiento tecnológico reduciendo su actividad notablemente. Ya no entregará netbooks al alumnado y plantel docente, eliminando el modelo 1:1 y planteando un equipamiento para la escuela de diferentes tipos de TIC (netbooks, diversos tipos de robots, etc.) que se utilicen dentro del espacio y horario escolar.

\section{Objetivos de investigación e hipótesis}

El objetivo general de este trabajo es identificar la influencia de la clase social en las representaciones sociales, apropiaciones, evaluación del PCI y consumos digitales y mediáticos.

Los objetivos específicos se corresponden con las siguientes tareas que se realizarán e indagarán en este trabajo. En primer lugar, el objetivo es configurar una tipología de alumnos beneficiarios del PCI y ubicar dichos tipos 
en el espacio social delimitado por las variables elegidas a tal fin en nuestro modelo de análisis. En segundo lugar, nos disponemos a identificar similitudes y diferencias en los grupos creados en relación a los consumos mediáticos y digitales, las representaciones sociales y evaluaciones del PCI. Asimismo este objetivo comprende el dar cuenta de la relación entre la disposición de los grupos en el campo social y la relación del mismo con la posición social de los individuos. Finalmente un tercer objetivo específico es detectar regularidades en los grupos y generar resultados sobre ellos que permitan avanzar en el desarrollo del objetivo general.

Las hipótesis de trabajo son:

- El nivel socioeconómico es un indicador de la relación del individuo con la tecnología.

- Las representaciones de los estudiantes sobre el PCI se relacionan con el buen o mal funcionamiento del mismo en la escuela a la cual concurre el alumno, la disponibilidad de TIC en el hogar y el nivel socioeconómico.

- Para alumnos de bajo nivel socioeconómico, el acceso en el hogar a las TIC es bajo, por lo que sus representaciones sobre el PCI se relacionan con el tener el objeto netbook, su primera computadora.

- Existen diferencias por sexo, teniendo un mayor desinterés y peores representaciones sociales sobre sí mismas en las mujeres. 


\section{Metodología y modelo de análisis}

Para realizar este trabajo utilizamos como fuente secundaria la base de datos recabada en el proyecto "Flujos de conocimientos, tecnologías digitales y actores sociales en la educación secundaria. Un análisis socio-técnico de las capas del Programa Conectar Igualdad" del Ministerio de Ciencia Tecnología e Innovación Productiva de la Nación; investigación liderada por el Dr. Mariano Zukerfeld y realizada en el año 2015. Esta base encuestó a 3183 alumnos de 23 escuelas elegidas de forma aleatoria de un total de 6140 secundarias de gestión estatal, urbanas y de educación común de la República Argentina, controlando por jurisdicción y departamento para conservar la heterogeneidad geográfica. La unidad de análisis es alumno/alumna de escuela media de gestión pública perteneciente a la República Argentina durante el año 2015, de primero a séptimo año dependiendo de la modalidad y el régimen provincial de la escuela. Las edades de los estudiantes varían entre los 12 y 34 años, teniendo el 96,4\% de los casos entre 13 y 19 años. En cuanto al turno, se incluyeron los turnos mañana, tarde y noche, representando este último al 2,4\% de los estudiantes. Las escuelas en las cuales se realizó esta encuesta corresponden a las provincias de Santa Fé, Córdoba, Misiones, Chaco, Corrientes, Mendoza, Entre Ríos, Tucumán, Santiago del Estero, Buenos Aires y a la Ciudad Autónoma de Buenos Aires, abarcando 10 de las 24 provincias más la ciudad capital.

Se realizó un modelo de análisis que considera tres dimensiones de la actividad del alumnado en relación al PCI. Una primera dimensión, relativa al nivel socioeconómico en la cual utilizamos las variables de nivel de estudios alcanzado por el adulto con quien el estudiante vive, situación ocupacional del adulto, si el alumno trabaja o trabajó alguna vez, y el estrato de la escuela a la cual el alumno concurre. Al tratarse los estratos, se siguió la clasificación aportada por el informe de la investigación "Flujos de conocimientos, tecnologías digitales y actores sociales en la educación secundaria. Un análisis socio-técnico de las capas del Programa Conectar Igualdad" que identifica 4 estratos dependiendo del acceso 
a recursos de infraestructura como a las tecnologías digitales (acceso alto, medio alto, medio bajo y bajo). Otra dimensión utilizada es la de acceso a la tecnología, considerando las variables de cuántas computadoras tiene el alumno en su casa y si la escuela tiene internet. La última dimensión agrupa las variables que indagan las representaciones sociales de los alumnos sobre las TIC, el PCI y la habilidad digital de actores escolares. Las variables de esta dimensión son: persona a quien consulta sobre dificultades con la netbook, evaluación de habilidad digital de docentes y alumnos, evaluación de las netbooks del PCI y representaciones (indagado como asociaciones) sobre el PCI. La variable sexo ha sido utilizada como una variable complementaria en el análisis.

\section{Tabla I}

Modelo de análisis

\begin{tabular}{|c|c|c|}
\hline Dimensión & Variable & Categorías \\
\hline \multirow{15}{*}{$\begin{array}{c}\text { Nivel } \\
\text { Socio- } \\
\text { económico }\end{array}$} & \multirow{4}{*}{$\begin{array}{c}\text { Situación laboral } \\
\text { del adulto }\end{array}$} & 1 Trabaja \\
\hline & & 2 Desocupado \\
\hline & & 3 Trabaja a veces \\
\hline & & $4 \mathrm{Ns} / \mathrm{nc}$ \\
\hline & \multirow{2}{*}{$\begin{array}{c}\text { Situación laboral } \\
\text { del alumno }\end{array}$} & 1 Trabaja o trabajó \\
\hline & & 2 No trabaja \\
\hline & \multirow{5}{*}{$\begin{array}{l}\text { Nivel educativo del } \\
\text { adulto }\end{array}$} & 1 Primario Incompleto \\
\hline & & 2 Primario \\
\hline & & 3 Secundario \\
\hline & & 4 Superior \\
\hline & & $5 \mathrm{~ns} / \mathrm{nc}$ \\
\hline & \multirow{4}{*}{ Estrato escolar } & 1 Acceso alto \\
\hline & & 2 Acceso medio alto \\
\hline & & 3 Acceso medio bajo \\
\hline & & 4 Acceso bajo \\
\hline
\end{tabular}




\begin{tabular}{|c|c|c|}
\hline \multirow{8}{*}{ Acceso a TIC } & \multirow{5}{*}{$\begin{array}{l}\text { Cantidad de } \\
\text { computadoras }\end{array}$} & 1 Ninguna \\
\hline & & 2 1pcPCI (solo la del PCI) \\
\hline & & $3+2$ pcPCI (más de 2 con la PCI) \\
\hline & & 4 1pcNOPCI (una que no es la del PCI) \\
\hline & & $5+2$ pcNOPCI (más de dos, no del PCI) \\
\hline & \multirow{3}{*}{$\begin{array}{l}\text { Internet en la } \\
\text { escuela }\end{array}$} & 1 INTnunca \\
\hline & & 2 INTaveces \\
\hline & & 3 INTsiempre \\
\hline \multirow{24}{*}{ Percepciones } & \multirow{4}{*}{ Consulta sobre TIC } & $1 \mathrm{C}$ Docente \\
\hline & & 2 C Alumno \\
\hline & & 3 C Referente \\
\hline & & $4 \mathrm{C}$ Otros \\
\hline & \multirow{4}{*}{$\begin{array}{l}\text { Habilidades } \\
\text { Docentes }\end{array}$} & 1 DocenteMal \\
\hline & & 2 DocenteRegular \\
\hline & & 3 DocenteBien \\
\hline & & 4 DocenteExcelente \\
\hline & \multirow{4}{*}{$\begin{array}{c}\text { Habilidades } \\
\text { Alumnos }\end{array}$} & 1 AlumMal \\
\hline & & 2 AlumRegular \\
\hline & & 3 AlumBien \\
\hline & & 4 AlumExcelente \\
\hline & \multirow{5}{*}{$\begin{array}{c}\text { Evaluación de las } \\
\text { netbooks }\end{array}$} & 1 NETMuy buenas \\
\hline & & 2 NETMalas \\
\hline & & 3 NETRegulares \\
\hline & & 4 NETBuenas \\
\hline & & 5 NETns/nc \\
\hline & \multirow{7}{*}{ Asociación del PCI } & 1 Con la netbook \\
\hline & & 2 PCIFaceJuegos \\
\hline & & 3 PCIinternet \\
\hline & & 4 PCIaudiovisual \\
\hline & & 5 PCIescolar \\
\hline & & 6 PCIotros \\
\hline & & 7 Con nada \\
\hline \multirow{2}{*}{ Sexo } & \multirow{2}{*}{ Sexo } & 1 Masculino \\
\hline & & 2 Femenino \\
\hline
\end{tabular}

Fuente: elaboración propia 
Es menester aclarar que las categorías fueron las mismas dispuestas en la base de datos utilizadas, a excepción de dos casos. En primer lugar, las categorías de la variable "educación del adulto con quien el alumno vive" fueron agrupadas por nivel completo, y en segundo lugar, "la percepción (analizada teóricamente como representaciones sociales) de Habilidades con TIC de Docentes y Alumnos" fue relevada en la encuesta utilizando una escala de 1 a 10. A fines metodológicos y dado que en análisis anteriores dentro de la variable sobre Habilidad del Alumno con TIC, los valores del 1 al 5 fueron asignados aleatoriamente ("random assign") por el programa SPAD ya que representan pocos casos, dispusimos crear cuatro categorías para dicha variable: Mal (1 a 3), Regular (4 a 6) , Bien (7 y 8) y Excelente ( 9 y 10). Dado que la misma escala de 1 a 10 se utilizaba para la variable Habilidad del Docente, decidimos realizar la misma operación y crear estas cuatro categorías a fin de que las mismas resulten comparables.

Con este modelo de análisis se estandarizaron los datos mediante el programa IBM SPSS Statistics para luego comenzar el análisis en el programa SPAD.

Se realizó un Análisis de Correspondencias Múltiples (ACM) para analizar las múltiples relaciones entre las variables cualitativas elegidas. En primer lugar, el Análisis Factorial nos permitió sintetizar la información, partiendo de un total de 49 categorías distribuidas en 12 variables, hasta llegar a la reducción de dos factores o ejes a partir de los cuales las mismas se distribuirán en el espacio social. Este tipo de análisis permite describir interrelaciones y asociar variables que se reflejan en este espacio social, representado por un gráfico con dos ejes X e Y (López-Roldán \& Fachelli, 2016). En un segundo momento el ACM combina el Análisis Factorial con el Análisis de Clasificación para construir tipologías, de esta manera "las variables factoriales se considerarán como las variables-criterio con las que clasificar las unidades en grupos homogéneos" (López-Roldán \& Fachelli, 2016: 157). De esta forma los individuos analizados se ubican en el espacio social construyendo 5 tipos definidos por la conjunción de variables ingresadas en el análisis. Una tercera etapa final es la validación de los resultados en la que las decisiones estadísticas se combinan con decisiones conceptuales relacionadas al modelo de análisis propuesto (Rodríguez de la 
Fuente, 2014: 4). Las características de cada tipo se desprenden del grado de contribución de cada categoría en la definición de la misma. A partir de estas contribuciones se han nombrado los grupos formados, intentando dar cuenta de las categorías principales presentes en la clasificación.

\section{Resultados del análisis}

Habiendo realizado el ACM se eliminaron algunas categorías activas con poco peso, partiendo de 11 variables con 48 categorías se redujo la cantidad a 11 variables con 45 categorías. En cuanto a los aspectos técnicos del análisis, se identifica una varianza de 53,39\% para el primer factor y 19,88\% para el segundo factor, obteniendo así un total de 73,27\% de varianza explicada en este trabajo. Al retener solo dos factores conseguimos ser parsimoniosos en el análisis e incluso simplificar la lectura de los resultados a fin de hacer más comunicables los hallazgos de este estudio.

Los resultados se presentan visualmente de dos formas, en el gráfico 1 presentamos los factores resultantes en los ejes X e Y, y la ubicación de cada variable en el espacio multidimensional definido. En el gráfico 2 se ubican los tipos conformados dentro de este espacio, identificando con diferentes colores los casos/individuos en los 5 grupos conformados. La composición de cada factor o eje se puede observar en la tabla II del anexo y a continuación realizaremos una breve descripción de los mismos. 


\section{Gráfico 1}

Categorías ubicadas en el espacio social

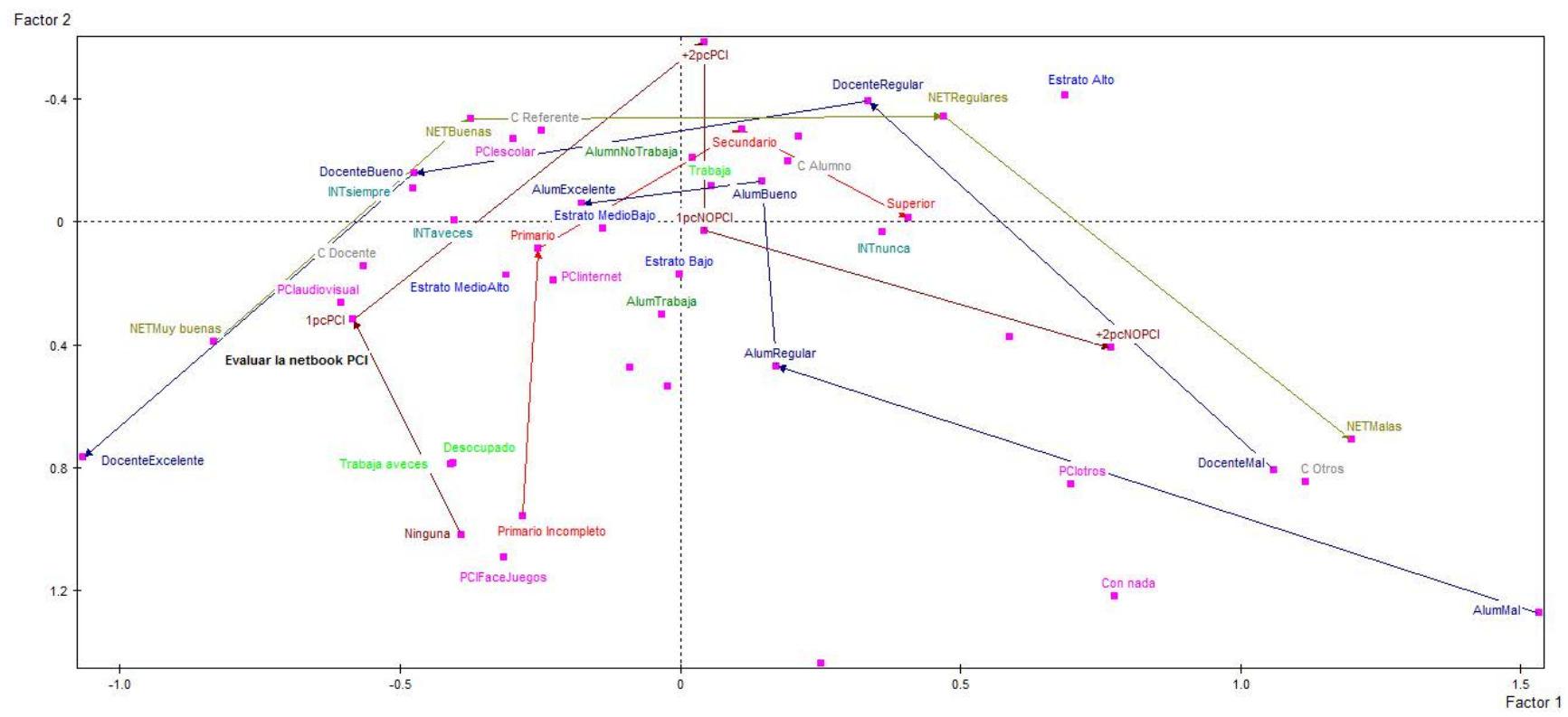

Fuente: elaboración propia

Los dos factores resultantes del Análisis de Correspondencias Múltiples fueron nombrados a partir de las variables que mayor proyección tienen en el eje que soporta dicho factor ( $\mathrm{X}$ o Y ), las cuales se pueden observar en el gráfico 1 a partir de la cercanía o lejanía de cada variable con respecto al eje nombrado.

Factor 1: Representación PCI. Este eje se corresponde con las representaciones sociales de los alumnos en relación al PCI, la percepción de habilidades de docentes y alumnos y la evaluación de las netbooks del PCI, así como también con qué relacionan al PCI. Las variables que se proyectan en este factor son 6: la calificación de las netbooks, las percepciones de habilidad TIC de docentes y alumnos, a quien consulta el alumno cuando no sabe hacer algo con la netbook, con que relacionan el Plan Conectar Igualdad y la existencia de conexión a internet en la escuela. Si bien la variable de internet podría parecer a primera vista parte del nivel socioeconómico es menester recordar que se trata de la conexión en la escuela, lo cual es parte del funcionamiento del PCI y no una cualidad del individuo particular. 
Factor 2: Nivel Socioeconómico. El factor dos se corresponde con las variables relativas al nivel socioeconómico del alumno. Las variables que se proyectan en este eje son cinco: la educación del adulto con quien vive; si el alumno trabaja; la situación laboral del adulto con quien vive; el estrato de la escuela a la que concurre; y la cantidad de computadoras que posee en el hogar.

Estos dos factores se corresponden con los dos conceptos centrales planteados teóricamente en el apartado de antecedentes: representaciones sociales y estructura social.

Tras obtener los dos factores entre los cuales se ordenarán las variables, se realizó el análisis de clasificación bajo el método jerárquico WARD que "consiste en un proceso progresivo de agregación de las unidades/grupos de manera que en cada etapa se unan aquellos dos elementos que supongan la mínima pérdida de inercia (o varianza)" (Lopez-Roldán \& Fachelli, 2016b: 35) configurando de esta forma grupos más homogéneos internamente y más heterogéneos entre sí. De esta manera quedaron definidos los 5 tipos en los cuales se ubican los casos analizados. La disposición de dichos casos ofrece una forma de curvatura en forma de arco denominado el efecto Guttman, lo cual pone en evidencia un cierto orden de las categorías.

Estos cinco tipos bajo los cuales se agruparon los casos de la muestra estudiada han sido nombrados bajo dos consignas centrales: la oposición incluidos / excluidos del PCI, y la estructura social a partir de clases sociales, identificando entre alta, media (solo incluidos) y baja. Es menester aclarar que las categorías "incluidos y excluidos" se refiere al funcionamiento de Conectar Igualdad en el instituto educativo al cual los estudiantes concurren, en términos de inclusión digital: la disponibilidad de servicio de internet $y$ el estrato de la escuela (que incluye acceso a TIC e infraestructura del establecimiento). La composición de los tipos finales se puede leer en la tabla III del anexo. 


\section{Gráfico 2}

Tipos ubicados en el espacio social

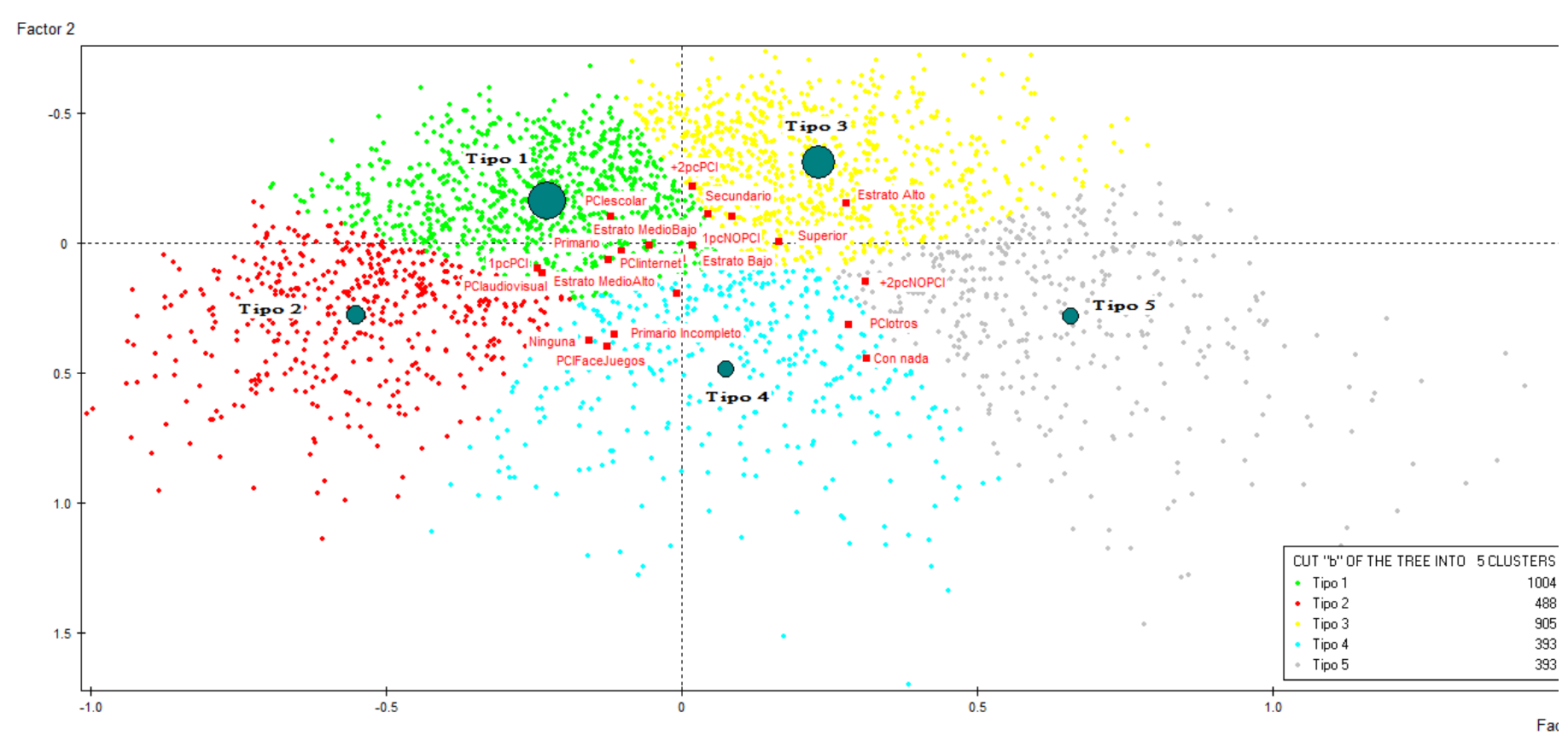

Fuente: elaboración propia

Tipo 1: Incluidos de clase media. El 31,54\% de los casos se ubica en este tipo. Este grupo se define como incluidos ya que en este grupo se ubican los alumnos que tienen un buen acceso a las TIC desde la escuela, gracias a la provisión de internet con buenos niveles (internet siempre y a veces) y a que la escuela a la que concurren es de los estratos medio alto y medio bajo.

En cuanto a las representaciones sociales, estos individuos tienen representaciones positivas sobre Conectar Igualdad, en relación a la habilidad docente (buena), la calidad de las netbooks (buenas) y las habilidades del alumnado (excelentes). La consulta cuando no saben realizar alguna tarea con la netbook es a los docentes, lo cual revela cierta confianza y valoración positiva en la institución educativa en torno a la tecnología. Los individuos de este grupo relacionan esta política con la actividad educativa. En cuanto a las características socioeconómicas son alumnos 
de padres ocupados que poseen dos computadoras o más en su casa, por lo cual se han clasificado como de clase media.

Tipo 2: Incluidos de clase baja. Este grupo concentra el 15,33\% de los casos. Este tipo es el grupo que tiene la mejor percepción del programa, docentes (excelente y bueno) y alumnos (excelente) con un alto nivel de habilidades digitales y califican a las netbooks como muy buenas. También pertenecen a escuelas con buen acceso, del estrato medio alto y con provisión de internet frecuente (a veces y siempre). Nuevamente las consultas sobre TIC se realizan a los docentes al igual que el tipo anterior. La identificación del PCI varía entre relacionarlo con usos lúdicos de redes sociales y juegos, también con usos audiovisuales de consumo, y también con la actividad escolar y con la conexión a internet. Este último punto sobre la conectividad podría ser decisivo en este grupo, ya que aparece en distintas variables que componen el tipo.

Elgrupo se compone de alumnos con hogares de bajos niveles educativos (primario o primario incompleto) y con situaciones laborales inestables (desocupados o que trabajan a veces). Muchos de estos alumnos han trabajado o trabajan, y algunos no tienen ninguna computadora en su hogar y otros cuentan con solo la netbook entregada por el PCI.

En futuros análisis cualitativos sería interesante rastrear en este tipo las relaciones entre la clase social de estos individuos, en relación a las restricciones de acceso a tecnología que podrían tener por su condición económica y el poder de compra de equipos, y las representaciones sociales sobre Conectar Igualdad, en cuanto una posibilidad de inclusión digital a partir de la posesión de la netbook como bien propio.

Tipo 3: Incluidos de clase alta. En este tipo se ordenan el 28,43\% de los casos. Los individuos de este grupo tienen percepciones regulares del PCI (regular) y de las netbooks (regular). La habilidad docente no aparece como variable definitoria para este tipo. 
En este grupo se encuentran algunas contradicciones en cuanto al acceso a TIC, si bien responden no tener nunca acceso a internet en la escuela, corresponden al grupo de escuelas del estrato alto y poseen en su hogar dos o más computadoras de las cuales una es la netbook del PCI. Relacionan al programa con la netbook que entrega.

Este tipo concentra individuos de hogares con alto nivel educativo (secundario y superior), adultos que trabajan y alumnos que nunca lo han hecho.

Al igual que en el tipo anterior, futuros análisis deberán puntualizar en las expectativas que este grupo tiene sobre Conectar Igualdad, la provisión de internet y la calidad de la netbook en relación a su clase social, en cuanto a la cantidad de computadoras, el acceso a la tecnología y a internet en sus hogares.

Este tipo tiene una fuerte presencia de mujeres, lo cual incluye la variable género, que se ha analizado como variable complementaria, dentro de este estudio.

Tipo 4: Excluidos de clase baja. El 12,35\% de los individuos pertenecen a este tipo. Este grupo es negativo o indiferente en cuanto al PCI: no lo relacionan con nada específico (las respuestas más frecuentes registradas son "no sabe no contesta" y "nada") o con redes sociales y juegos. Califican como malas las habilidades docentes (mal) y de los alumnos (regular y mal), al igual que las netbooks (mala). Muchos no tienen la netbook del PCI y ninguna otra computadora en el hogar, por lo cual no podrían evaluar la calidad de la netbook. Sobre este punto es importante aclarar que la base de datos utilizada no especifica si los alumnos nunca tuvieron la netbook o si en realidad la tuvieron y actualmente no está disponible, ya sea por rotura o por bloqueo: en los focus group que actualmente la autora está realizando, los estudiantes reconocen que estas dos causas son la razón por la cual no consideran poseer la netbook de Conectar Igualdad. 
Los alumnos de este grupo trabajaron o trabajan actualmente, provienen de hogares con bajo nivel educativo (primario incompleto) y adultos desocupados o que trabajan a veces, por lo cual se los englobó en la categoría de clase baja.

El estrato escolar que predomina en este tipo es el medio alto, sin embargo, parece representar a aquellos que están "afuera" del PCI, asociando el programa con la respuesta "otros" o "nada", con poco acceso a TIC (por la respuesta de cantidad de computadoras) y sin poseer la netbook.

Tipo 5: Excluidos de clase alta. Al igual que el grupo anterior, este concentra el $12,35 \%$ de los individuos. Los casos agrupados en este tipo tienen negativas asociaciones del PCI, no relacionan al programa con nada o respondieron "otros" al ser consultados, califican de malas las netbooks, las habilidades docentes, las habilidades del alumnado (regular y mal) y nunca tienen internet, a pesar de pertenecer al estrato escolar alto.

En cuanto a las características socioeconómicas los alumnos poseen dos o más computadoras en su hogar, pero ninguna es la netbook del PCI, el adulto responsable está ocupado y posee alto nivel educativo (superior), y los alumnos han trabajado o trabajan. Al igual que el tipo 4, este tipo podría representar a individuos excluidos del acceso propuesto por el PCI, pero con alumnos de un mayor nivel socioeconómico. Al igual que el grupo 3, las expectativas sobre el PCI deberían ser rastreadas en relación a la clase social y el acceso hogareño a TIC.

Hay una fuerte presencia masculina en este tipo. Si bien, el sexo ha sido una variable complementaria del Análisis de Correspondencias Múltiples, es interesante evidenciar que entre los grupos con presencia de esta dimensión, el femenino (grupo 3) tiene representaciones regulares del PCI mientras que el masculino (grupo 5) tiene representaciones más bien negativas del mismo. En este punto las 
expectativas y la relación entre género y tecnología serían caminos a indagar en análisis de tipo cualitativos.

\section{Conclusiones}

Como era de esperar, las dos dimensiones centrales de clases sociales y representaciones han sido los ejes bajo los cuales se creó la tipología, confirmando parte de la exposición teórica de este trabajo en los resultados estadísticos del análisis propuesto.

Tras realizar el Análisis de Correspondencias Múltiples y configurar los cinco tipos anteriormente descritos, se evidencia que las representaciones sociales sobre Conectar Igualdad están íntimamente relacionadas, por un lado, con el modo en que funciona el programa en la institución educativa a la cual pertenecen los estudiantes y, por otro, con el acceso a las TIC por parte de ellos. El primer punto estableció una clara diferenciación entre quienes están incluidos y quienes se encuentran excluidos del PCI (dónde funciona mejor y peor). Este aspecto, sin embargo, no es del todo visible en el tipo 3, donde encontramos diferentes variables que pueden contradecir el grado de acceso como, por ejemplo, no tener nunca internet pero si poseer la netbook y pertenecer a una escuela del estrato alto de acceso a TIC. Este grupo tiene una representación regular del PCI. Comparándolo con los tipos 1 y 2 que están incluidos en el PCI observamos que a medida que baja el nivel socioeconómico aumenta la representación positiva del programa, siendo los alumnos del tipo 2 incluidos de clase baja los más positivos con respecto al programa y los del tipo 3 incluidos de clase alta los más reticentes al mismo. De esta forma, observamos la emergencia del segundo punto que refiere a la posibilidad de acceder a tecnología de los estudiantes individualmente. Es así, que dentro de los excluidos, quienes pueden tener acceso a TIC y quienes no poseen otra computadora y tienen un menor nivel socioeconómico (lo cual les dificulta estar incluidos digitalmente en forma individual) tienen representaciones más positivas sobre Conectar Igualdad que quienes cuentan con otras computadoras y tienen mejores posiciones socioeconómicas. En estos últimos casos, la implementación del PCI será fundamental para permitir la inclusión de grupos que de otra forma 
estarían excluidos digitalmente, como ocurre con el tipo 2 de incluidos de clase baja. Por otro lado, quienes más acceso tendrían en términos socioeconómicos, tienen peores representaciones sobre el PCI. La apatía, rechazo o representaciones negativas sobre el PCI en estos grupos, si bien son similares a partir del análisis estadístico, requerirán en un futuro un análisis cualitativo más profundo que de cuenta de las diferentes razones de estas actitudes, las cuales parecen provenir de la posición en la estructura social de los individuos (la clase social) en cuanto a posibilidades de acceso a TIC y representaciones sociales sobre el funcionamiento de la tecnología.

En este sentido, también encontramos que otros aspectos relacionados con Conectar Igualdad como las habilidades docentes o la calidad de las netbooks, se relacionan con el buen o mal funcionamiento del plan dentro de la escuela.

Como ya se explicó en las páginas anteriores, sería interesante trabajar en un futuro la relación entre la clase social y la representación social de inclusión digital como posibilidad. La hipótesis que guiaría esta línea de indagación es la siguiente: aquellos alumnos cuyo nivel socioeconómico les permite incluirse digitalmente de forma privada tienen una baja valoración del PCI y de la netbook entregada ya que posiblemente cuenten con recursos digitales mejores en sus hogares o tengan acceso a múltiples TIC, por lo tanto la inclusión digital sería un hecho en sus trayectorias digitales. Inversamente los alumnos con menos posibilidades de consumo y acceso a TIC reconocen en el PCI la potencialidad de incluirlos digitalmente y otorgarles un objeto TIC que de otra forma no sería posible obtener, planteando la inclusión digital como posibilidad.

El género no es una cuestión menor, si bien en el análisis fue tomado como una variable complementaria. En cuanto a las diferencias por sexo encontramos una mayor positividad de las mujeres (tipo 3, sexo con un test value de 3,20) y una negatividad de los hombres (tipo 5, sexo con un test value de 5,07), pero 
no podemos predecir si esta actitud se relaciona o no con el acceso a TIC y la inclusión en el PCI o no por pertenecer a dos tipos con diferentes grados de participación en el programa. En este sentido es menester plantear otra línea de investigación en un futuro que indague sobre las representaciones de género en torno a la tecnología. La representación social detrás de esta diferencia dispone a los hombres como "mejores", más capaces que las mujeres y con disposiciones más "naturales" para la tecnología. Siendo "algo más para varones", las mujeres demostrarían menos interés en TIC. Si estas representaciones son las que figuran en el análisis de los tipos 3 y 5 , podría argumentarse que las evaluaciones femeninas como "regulares" y de los varones como "malas" en cuanto a la netbook y a diferentes aspectos del PCI, se deben a cierta masculinización del campo tecnológico y una mayor exigencia o expectativas de los hombres en relación a las TIC. Si bien en este análisis cualitativo dichos resultados aparecen como diferencias, considero que esconden una desigualdad de género como se explicó más arriba, la cual se planea explorar en futuros trabajos a partir de los estudios feministas que plantean una segunda brecha digital en relación al género.

En cuanto a las hipótesis a comprobar, en primer lugar este trabajo comprobó la hipótesis que plantea la relación entre las representaciones sociales sobre el PCI y su funcionamiento, es decir, el nivel de inclusión o exclusión en el mismo. En menor medida se comprobó la relación de estas representaciones con el nivel socioeconómico de los alumnos. En segundo lugar, refutamos la hipótesis que planteaba la asociación de Conectar Igualdad al objeto netbook en las clases bajas. Los alumnos de bajo nivel socioeconómico incluidos en el PCI (tipo 2) relacionan al programa, mayormente, con actividades lúdicas, juegos y redes sociales, y en un segundo lugar, con la actividad escolar y la conectividad a internet. Este hallazgo nos resulta particularmente interesante para futuras investigaciones ya que la actividad lúdica podría estar relacionada con los consumos mediáticos del hogar de origen y las trayectorias digitales individuales a través de patrones familiares 
preexistentes.

Finalmente, el objetivo ulterior del presente artículo ha sido abrir preguntas a futuro y, a partir de identificar estos cinco tipos de usuarios/alumnos beneficiarios del PCI, avanzar en un análisis cualitativo de los tipos encontrados para acercarnos a responder a la pregunta de por qué individuos que podrían pertenecer a la misma clase social por su nivel socioeconómico (y también por su bagaje cultural, lo cual no ha sido analizado en este trabajo) tienen representaciones digitales diferentes y generan modos de apropiación distintos entre sí, diferencias que a primera vista no podemos observar con este tipo de análisis. Creemos que la respuesta se encuentra en analizar las trayectorias digitales de los estudiantes, prestando especial atención a la obtención del objeto netbook por parte del Plan Conectar Igualdad como un evento relevante, y también al dominio de la escuela que como institución educativa es también un eje central, ya que la experiencia escolar con tecnología del individuo podría incidir también en los modos de apropiación digital. 


\section{Bibliografía}

- AREA MOREIRA, M. (2014). Las políticas educativas TIC en España después del Programa Escuela 2.0: las tendencias que emergen. RELATEC, Revista Latinoamericana de Tecnología Educativa, 13 (2), 11 - 33.

- ARTOPOULOS, A. \& KOZAK, D. (2011). Topografías de la integración de TIC en Latinoamérica. Hacia la interpretación de los estilos de adopción de tecnología en educación. Buenos Aires: Universidad de San Andrés, Centro de Tecnología y Sociedad, Documento de trabajo $N^{\circ}$ 9. Extraído de www.udesa.edu.ar/files/ AdmTecySociedad/DT\%209.pdf

- BENÍTEZ LARGHI, S. (2017). Desigualdades 2.0: un estudio cualitativo sobre los vínculos entre desigualdad social y desigualdad digital. Ponencia presentada en el IV Seminario Internacional de Desigualdad y Movilidad Social. Facultad de Humanidades y Ciencias de la Educación, Universidad de La Plata. La Plata: UNLP.

- BENÍTEZ LARGHI, S., LEMUS, M., MOGUILLANSKY, M. \& WELSCHINGER LASCANO, N. (2015). Digital and Social Inequalities: A Qualitative Assessment of the Impact of the Connecting Equality Program on Argentinean Youth. The Electronic Journal of Information Systems in Developing Countries, 69, 1-20. s.l.:EJISDC.

- BOURDIEU, P. (1979). La distinción. Criterios y bases sociales del gusto. México: Taurus.

- BUCKINGHAM, D. (2008). Más Allá de la tecnología: aprendizaje infantil en la era de la cultura digital. Buenos Aires: Manantial.

- CAMACHO, K. (2005). La brecha digital. En A. Ambrosi et al. (coord.), Palabras en juego: Enfoques Multiculturales sobre las Sociedades de la Información. Caen: C\&F Éditions.

- CASTELLS, M. (1997). La era de la información. Economía, sociedad y cultura Vol. I. México DF: Siglo XXI.

- CEPAL (Comisión Económica para América Latina y el Caribe). (2003). Los caminos hacia una sociedad de la información en América Latina y el Caribe, LC/G.2195/Rev. 1- P, Santiago de Chile: CEPAL. 
- Decreto 459/10 - EDUCACIÓN - Crea Programa "Conectar Igualdad.Com. Ar" de incorporación de la nueva tecnología para el aprendizaje de alumnos y docentes. Consultado el 6/03/2018 en http://www.bnm.me.gov.ar/giga1/ normas/14691.pdf

- Decreto 386/2018 Creación del Plan Aprender Conectados. Consultado el 16/05/2018 en http://servicios.infoleg.gob.ar/infolegInternet/ anexos/305000-309999/309610/norma.htm

- DI MAGGiO, P., HARGiTTAI, E., CELESTE, C. \& SHAFER, S. (2001). From the 'Digital Divide' to 'Digital Inequality': Studying Internet access as penetration increases. Working Papers Series, (15). Princeton: Princeton University.

- DUGHERA, L. (2015). De Internet, computadoras portátiles, softwares y contenidos. Un análisis comparativo de planes "una computadora, un alumno" en tres provincias de la Argentina. (Tesis doctoral). Facultad Latinoamericana de Ciencias Sociales (FLACSO) sede Académica Buenos Aires, Buenos Aires, Argentina. Disponible en http://e-tcs.org/wp-content/uploads/2016/02/ Tesis-Doctoral-Dughera-Final-con-car\%C3\%A1tula.pdf

- FRAU MEIGS, D. (2011). El vínculo entre educación para los medios y derechos humanos: Una necesidad y una oportunidad. Derecho a Comunicar, 1, 173-190.

- FUNDACIÓN, S. (2013). Y las mujeres ¿dónde están? Estudio sobre representaciones acerca de la informática en escuelas secundarias del conurbanobonaerense (informe). Buenos Aires: Ministerio de Ciencia, Tecnología e Innovación Productiva, Argentina.

- GARCía CANCLINI, N. (1995). Consumidores y ciudadanos. Conflictos multiculturales de la globalización. México: Grijalbo.

- JODELET, D. (1986). La representación social: fenómenos, concepto y teoría. En S. Moscovici (comp.), Psicología social II. Pensamiento y vida social. Psicología social y problemas sociales. Barcelona: Paidós.

- LAGO MARTÍNEZ, S. (2012). Inclusión digital en la educación pública argentina. El Programa Conectar Igualdad. Revista Educación y Pedagogía, 24 (62), 205218. 
- LAGO MARTÍNEZ, S. (2015). De tecnologías digitales, educación formal y políticas públicas. Aportes al debate. Ciudad Autónoma de Buenos Aires: Editorial Teseo.

- LEVIS D., DIÉGUEZ, S. \& REY, E. (2011). Redes educativas 2.1. Medios sociales, entornos colaborativos y procesos de enseñanza y aprendizaje en RUSC. Revista de Universidad y Sociedad del Conocimiento, 8 (1), 7-24.

- Ley 27.078 ARGENTINA DIGITAL (Boletín Oficial № 33.034, 19/12/14) Consultada el 23/04/2018 en https://www.enacom.gob.ar/ley-27-078_p2707

- LÓPEZ-ROLDÁN, P. \& FACHELLI, S. (2016). Análisis factorial. En P. López-Roldán \& S. Fachelli (1a edición), Metodología de la Investigación Social Cuantitativa (pp. 6-13). Capítulo III.11. Versión 3 (octubre de 2016). Edición digital: http://ddd. uab.cat/record/142928. Bellaterra (Cerdanyola del Vallès): Dipòsit Digital de Documents, Universitat Autònoma de Barcelona, Barcelona.

- LÓPEZ-ROLDÁN, P. \& FACHELLI, S. (2016 b). Análisis de clasificación. En P. López-Roldán y S. Fachelli, Metodología de la Investigación Social Cuantitativa (pp. 4-109). Capítulo III.12. 1a edición. Versión 2. Edición digital: http://ddd. uab.cat/record/142929. Bellaterra (Cerdanyola del Vallès): Dipòsit Digital de Documents, Universitat Autònoma de Barcelona.

- MARTín BARBERO, J. (1987). De los medios a las mediaciones. Comunicación, cultura y hegemonía. Barcelona: Gustavo Gilli.

- MARTÍNEZ-REINA, M.C. \& VÉLEZ CEA, M. (2010). Estereotipos de género en el juego y en el ocio tecnológico interactivo. Toluca: Ciencia Ergo-sum, 16 (2).

- MORLEY, D. (1996). Interpretar televisión: la audiencia de Nationwide. En D. Morley. Televisión, audiencias y estudios culturales (pp. 111-147). Buenos Aires: Amorrortu.

- ORGANIZACIÓN DE ESTADOS AMERICANOS (OEA). (2011). Comunicado de prensa: Relatorías de libertad de expresión emiten declaración conjunta acerca de internet. Comunicado de prensa R50/11. Disponible en OEA R50/11. Recuperado el 21/03/2018 en http://www.oas.org/es/cidh/expresion/ showarticle.asp?artID $=848$ 
- PARSONS, T. (1954). Ensayos de teoría sociológica. Buenos Aires: Paidós.

- PLÁ, J. (2013). Acerca de las potencialidades del concepto de clase para el campo de estudios de la movilidad social. Aposta: Revista de ciencias sociales, 58, 7-29.

- RODRÍGUEZ DE LA FUENTE, J.J. (2014). La movilidad social en el espacio multidimensional: Ciudad Autónoma de Buenos Aires 2012-2013. Barcelona: Trabajos de investigación. Disponible en http://ddd.uab.cat/record/137800

- ROSS, C. (2014). Inclusión digital y prácticas de enseñanza en el marco del Programa Conectar Igualdad para la formación docente del nivel secundario, ROSS, C. (coord.) (2014). Ciudad Autónoma de Buenos Aires: Ministerio de Educación de la Nación.

- SELWYN, N. (2004). Reconsidering political and popular understandings of the digital divide. New Media \& Society, 6 (3), 341-362.

- TEDESCO, J.C. (Abril, 2005). Las TICs y la desigualdad educativa en América Latina. Presentado en el Tercer Seminario Las Tecnologías de Información y Comunicación y los Desafíos del Aprendizaje en la Sociedad del Conocimiento. Seminario llevado a cabo en el CEDI/OCDE de Habla Hispana entre el 30 de marzo y el 1 de abril, Santiago de Chile, Chile.

- THOMPSON, J. (1990). Ideología y cultura moderna, (Ed. 2006). México: Universidad Autónoma Metropolitana.

- VACCHIERI, A. (2013). Las políticas TIC en los sistemas educativos de América Latina. Caso Argentina en Programa TIC y Educación Básica. Buenos Aires: Fondo de las Naciones Unidas para la Infancia (UNICEF). Disponible en http://www. unicef.org/argentina/spanish/Argentina_ok.pdf

- VAN DEURSEN, A. J.A.M., HELSPER, E., EYNON, R. \& VAN DIJK, J. A.G.M. (2017). The compoundness and sequentiality of digital inequality. International Journal of Communication, 11, 452-473. 
- WEBER, M. (1964). Economía y sociedad. Madrid: Fondo de Cultura Económica.

- WINOCUR, R. (2009). Robinson Crusoe ya tiene celular: la conexión como espacio de control de la incertidumbre. México: Siglo XXI, Universidad Autónoma Metropolitana: Unidad Iztapalapa.

- WINOCUR, R. (2013). Una revisión crítica de la apropiación en la evaluación de los programas de inclusión digital. En S. Morales y M. Loyola (comps.), Nuevas perspectivas en los estudios de comunicación. La apropiación tecno-mediática (53-64). Buenos aires: Imago Mundi.

- ZUKERFELD, M. (2010). Capitalismo y Conocimiento: Materialismo Cognitivo, Propiedad Intelectual y Capitalismo Informacional (Tesis doctoral). Volumen II "Capitalismo y Conocimiento" y Volumen III "Las regulaciones del Acceso a los conocimientos en el Capitalismo Informacional: Propiedad Intelectual y más allá". Facultad Latinoamericana de Ciencias Sociales (FLACSO) sede Académica Buenos Aires, Argentina.

- ZUKERFELD, M. (2015). Flujos de conocimientos, tecnologías digitales y actores sociales en la educación secundaria. Un análisis socio-técnico de las capas del Programa Conectar Igualdad (Informe Final). CIECTI 01_06_33. 


\section{Biografía}

\section{Victoria Matozo}

Instituto de Investigaciones Gino Germani Universidad de Buenos Aires (UBA) Consejo Nacional de Investigaciones Científicas y Técnicas (CONICET) victoria.matozo@gmail.com

Licenciada en Ciencias de la Comunicación Social (UBA), profesora en enseñanza media y superior en Comunicación Social (UBA). Becaria Doctoral del Consejo Nacional de Investigaciones Científicas y Técnicas (CONICET) de Argentina. Actualmente realiza el Doctorado en Ciencias Sociales en la Universidad de Buenos Aires (UBA) y la Diplomatura de postgrado de Metodología para el Análisis de las Desigualdades Sociales (MADES) en la Universidad Autónoma de Barcelona auspiciado por International Network for Comparative Analysis of Social Inequalities (INCASI).

Participa en el equipo Desigualdad y Movilidad Social dirigido por Eduardo Chávez Molina en el Instituto de Investigaciones Gino Germani desde el año 2015 realizando trabajos sobre análisis del discurso político, escolar y académico, educación y la inclusión de TIC (Tecnologías de la Información y la Comunicación), Juventud y Tecnología, Planes 1:1 de tecnología educativa y otras temáticas afines a desigualdad y educación. 


\section{Anexo}

Tabla II

Contribuciones de las categorías a cada eje

\begin{tabular}{|c|c|c|c|c|}
\hline Etiqueta & Peso relativo & $\begin{array}{l}\text { Distancia al } \\
\text { origen }\end{array}$ & Eje 1 & Eje 2 \\
\hline \multicolumn{5}{|l|}{ Internet en escuela } \\
\hline INTnunca & 4,932 & 0,84308 & 0,36 & 0,03 \\
\hline INTaveces & 2,730 & 2,32950 & $-0,40$ & 0,00 \\
\hline INTsiempre & 1,428 & 5,36600 & $-0,48$ & $-0,11$ \\
\hline \multicolumn{5}{|c|}{ Evaluar la netbook PCI } \\
\hline NETMuy buenas & 1,637 & 4,55497 & $-0,83$ & 0,39 \\
\hline NETMalas & 0,963 & 8,44510 & 1,20 & 0,71 \\
\hline NETRegulares & 2,762 & 2,29162 & 0,47 & $-0,34$ \\
\hline NETBuenas & 3,242 & 1,80441 & $-0,37$ & $-0,33$ \\
\hline NETns/nc & 0,488 & 17,61400 & 0,25 & 1,43 \\
\hline \multicolumn{5}{|c|}{ Alumno trabaja o trabajó } \\
\hline AlumTrabaja & 3,687 & 1,46553 & $-0,03$ & 0,30 \\
\hline AlumnNoTrabaja & 5,404 & 0,68235 & 0,02 & $-0,21$ \\
\hline \multicolumn{5}{|l|}{ EduAdulAgrupado } \\
\hline Primario Incompleto & 0,600 & 14,15710 & $-0,28$ & 1,00 \\
\hline Primario & 3,587 & 1,53424 & $-0,25$ & 0,09 \\
\hline Secundario & 3,065 & 1,96645 & 0,11 & $-0,30$ \\
\hline Superior & 1,839 & 3,94255 & 0,40 & 0,00 \\
\hline
\end{tabular}




\begin{tabular}{|c|c|c|c|c|}
\hline \multicolumn{5}{|l|}{ ConsultaTICok } \\
\hline C Docente & 3,016 & 2,01420 & $-0,57$ & 0,14 \\
\hline C Alumno & 4,890 & 0,85923 & 0,19 & $-0,20$ \\
\hline C Referente & 0,411 & 21,10420 & $-0,25$ & $-0,30$ \\
\hline C Otros & 0,774 & 10,74540 & 1,12 & 0,85 \\
\hline \multicolumn{5}{|c|}{ Cantidad computadoras en hogar } \\
\hline Ninguna & 0,940 & 8,67477 & $-0,39$ & 1,02 \\
\hline $1 \mathrm{pcPCI}$ & 1,651 & 4,50692 & $-0,58$ & 0,32 \\
\hline$+2 \mathrm{pcPCI}$ & 3,622 & 1,51025 & 0,04 & $-0,59$ \\
\hline $1 \mathrm{pcNOPCI}$ & 1,431 & 5,35329 & 0,04 & 0,03 \\
\hline$+2 \mathrm{pcNOPCI}$ & 1,448 & 5,27811 & 0,77 & 0,41 \\
\hline \multicolumn{5}{|c|}{ ¿Con qué cosas de la lista asocias al PCI? } \\
\hline Con la netbook & 3,864 & 1,35255 & 0,21 & $-0,28$ \\
\hline PCIFaceJuegos & 0,617 & 13,73610 & $-0,31$ & 1,09 \\
\hline PCIinternet & 1,000 & 8,09429 & $-0,23$ & 0,19 \\
\hline PCIaudiovisual & 0,346 & 25,30580 & $-0,60$ & 0,26 \\
\hline PCIescolar & 2,511 & 2,62116 & $-0,30$ & $-0,27$ \\
\hline PCIotros & 0,326 & 26,92110 & 0,70 & 0,85 \\
\hline Con nada & 0,428 & 20,22000 & 0,77 & 1,22 \\
\hline \multicolumn{5}{|l|}{ LaboralAdultoOK } \\
\hline Trabaja & 7,948 & 0,14373 & 0,05 & $-0,11$ \\
\hline Desocupado & 0,554 & 15,40720 & $-0,37$ & 0,81 \\
\hline Trabaja aveces & 0,588 & 14,45150 & $-0,38$ & 0,78 \\
\hline \multicolumn{5}{|c|}{ EstratosAgrupados } \\
\hline Estrato Alto & 1,816 & 4,00472 & 0,69 & $-0,41$ \\
\hline Estrato MedioAlto & 2,485 & 2,65862 & $-0,31$ & 0,17 \\
\hline Estrato MedioBajo & 3,439 & 1,64369 & $-0,14$ & 0,02 \\
\hline Estrato Bajo & 1,351 & 5,72939 & 0,00 & 0,17 \\
\hline \multicolumn{5}{|c|}{ HabAlumnoAgupado } \\
\hline AlumMal & 0,226 & 39,29110 & 1,53 & 1,27 \\
\hline AlumRegular & 0,825 & 10,01380 & 0,17 & 0,47 \\
\hline AlumBueno & 2,885 & 2,15149 & 0,15 & $-0,13$ \\
\hline AlumExcelente & 5,155 & 0,76344 & $-0,18$ & $-0,06$ \\
\hline
\end{tabular}




\section{HabDocenteAgrupado}

\begin{tabular}{|l|c|c|c|c|}
\hline DocenteMal & 1,357 & 5,70105 & 1,06 & 0,81 \\
\hline DocenteRegular & 3,507 & 1,59202 & 0,34 & $-0,39$ \\
\hline DocenteBueno & 3,202 & 1,83943 & $-0,47$ & $-0,16$ \\
\hline DocenteExcelente & 1,025 & 7,86629 & $-1,07$ & 0,77 \\
\hline
\end{tabular}

\section{Cargas de las categorías activas}

\begin{tabular}{|l|c|c|c|c|}
\hline Etiqueta & Peso relativo & $\begin{array}{c}\text { Distancia al } \\
\text { origen }\end{array}$ & Eje 1 & Eje 2 \\
\hline
\end{tabular}

\section{Internet en escuela}

\begin{tabular}{|l|c|c|c|c|}
\hline INTnunca & 4,932 & 0,84308 & 0,36 & 0,03 \\
\hline INTaveces & 2,730 & 2,32950 & $-0,40$ & 0,00 \\
\hline INTsiempre & 1,428 & 5,36600 & $-0,48$ & $-0,11$ \\
\hline
\end{tabular}

\section{Evaluar la netbook PCI}

\begin{tabular}{|l|c|c|c|c|}
\hline NETMuy buenas & 1,637 & 4,55497 & $-0,83$ & 0,39 \\
\hline NETMalas & 0,963 & 8,44510 & 1,20 & 0,71 \\
\hline NETRegulares & 2,762 & 2,29162 & 0,47 & $-0,34$ \\
\hline NETBuenas & 3,242 & 1,80441 & $-0,37$ & $-0,33$ \\
\hline NETns/nc & 0,488 & 17,61400 & 0,25 & 1,43 \\
\hline Alumno trabaja o trabajó \\
\hline AlumTrabaja & 3,687 & 1,46553 & $-0,03$ & 0,30 \\
\hline AlumnNoTrabaja & 5,404 & 0,68235 & 0,02 & $-0,21$ \\
\hline EduAdulAgrupado & 0,600 & 14,15710 & $-0,28$ & 1,00 \\
\hline Primario Incompleto & 3,587 & 1,53424 & $-0,25$ & 0,09 \\
\hline Primario & 3,065 & 1,96645 & 0,11 & $-0,30$ \\
\hline Secundario & 1,839 & 3,94255 & 0,40 & 0,00 \\
\hline Superior & 3,016 & 2,01420 & $-0,57$ & 0,14 \\
\hline ConsultaTICok & 4,890 & 0,85923 & 0,19 & $-0,20$ \\
\hline C Docente & 0,411 & 21,10420 & $-0,25$ & $-0,30$ \\
\hline C Alumno & 0,774 & 10,74540 & 1,12 & 0,85 \\
\hline C Referente
\end{tabular}




\section{Cantidad computadoras en hogar}

\begin{tabular}{|l|c|c|c|c|}
\hline Ninguna & 0,940 & 8,67477 & $-0,39$ & 1,02 \\
\hline 1 pcPCI & 1,651 & 4,50692 & $-0,58$ & 0,32 \\
\hline+2 pcPCI & 3,622 & 1,51025 & 0,04 & $-0,59$ \\
\hline 1 pcNOPCI & 1,431 & 5,35329 & 0,04 & 0,03 \\
\hline+2 pcNOPCI & 1,448 & 5,27811 & 0,77 & 0,41 \\
\hline
\end{tabular}

¿Con qué cosas de la lista asocias al PCI?

\begin{tabular}{|l|c|c|c|c|}
\hline Con la netbook & 3,864 & 1,35255 & 0,21 & $-0,28$ \\
\hline PCIFaceJuegos & 0,617 & 13,73610 & $-0,31$ & 1,09 \\
\hline PCIinternet & 1,000 & 8,09429 & $-0,23$ & 0,19 \\
\hline PCIaudiovisual & 0,346 & 25,30580 & $-0,60$ & 0,26 \\
\hline PCIescolar & 2,511 & 2,62116 & $-0,30$ & $-0,27$ \\
\hline PCIotros & 0,326 & 26,92110 & 0,70 & 0,85 \\
\hline Con nada & 0,428 & 20,22000 & 0,77 & 1,22 \\
\hline LaboralAdultoOK & 7,948 & 0,14373 & 0,05 & $-0,11$ \\
\hline Trabaja & 0,554 & 15,40720 & $-0,37$ & 0,81 \\
\hline Desocupado & 0,588 & 14,45150 & $-0,38$ & 0,78 \\
\hline Trabaja a veces & 1,816 & 4,00472 & 0,69 & $-0,41$ \\
\hline EstratosAgrupados & 2,485 & 2,65862 & $-0,31$ & 0,17 \\
\hline Estrato Alto & 3,439 & 1,64369 & $-0,14$ & 0,02 \\
\hline Estrato MedioAlto & 1,351 & 5,72939 & 0,00 & 0,17 \\
\hline Estrato MedioBajo & \multicolumn{5}{|l|}{} \\
\hline Estrato Bajo & 0,226 & 39,29110 & 1,53 & 1,27 \\
\hline HabAlumnoAgupado & 0,825 & 10,01380 & 0,17 & 0,47 \\
\hline AlumMal & 2,885 & 2,15149 & 0,15 & $-0,13$ \\
\hline AlumRegular & 5,155 & 0,76344 & $-0,18$ & $-0,06$ \\
\hline AlumBueno & 1,357 & 5,70105 & 1,06 & 0,81 \\
\hline AlumExcelente & 3,507 & 1,59202 & 0,34 & $-0,39$ \\
\hline HabDocenteAgrupado & 3,202 & 1,83943 & $-0,47$ & $-0,16$ \\
\hline DocenteMal & 1,025 & 7,86629 & $-1,07$ & 0,77 \\
\hline DocenteRegular & \multicolumn{5}{|l|}{} \\
\hline DocenteBueno & \multicolumn{5}{|l|}{} \\
\hline DocenteExcelente & \multicolumn{5}{|l|}{} \\
\hline
\end{tabular}

Fuente: elaboración propia 


\section{Tabla III}

Caracterización por categorías de cada tipo

\section{Tipo 1 (Casos: 1004 Porcentaje: 31.54 )}

\begin{tabular}{|l|c|c|}
\hline Etiqueta de la Variable & Categorías Caracteristicas & Test-value \\
\hline HabDocenteAgrupado & DocenteBueno & 16,91 \\
\hline Evaluar la netbook PCI & NETBuenas & 16,12 \\
\hline ¿Con qué cosas de la lista asocias al PCI? & PCIescolar & 7,93 \\
\hline ConsultaTICok & C Docente & 7,90 \\
\hline Internet en escuela & INTsiempre & 7,44 \\
\hline Internet en escuela & INTaveces & 6,83 \\
\hline Cantidad computadoras en hogar & +2pcPCI & 6,64 \\
\hline HabAlumnoAgupado & AlumExcelente & 6,36 \\
\hline EstratosAgrupados & Estrato MedioBajo & 5,30 \\
\hline LaboralAdultoOK & Trabaja & 3,95 \\
\hline EduAdulAgrupado & Primario & 3,69 \\
\hline EstratosAgrupados & Estrato MedioAlto & 3,24 \\
\hline
\end{tabular}

Tipo 2 (Casos: 488 Porcentaje: 15.33)

\begin{tabular}{|l|c|c|}
\hline Etiqueta de la Variable & Categorías Caracteristicas & Test-value \\
\hline HabDocenteAgrupado & DocenteExcelente & 21,64 \\
\hline Evaluar la netbook PCI & NETMuy buenas & 19,28 \\
\hline ConsultaTICok & C Docente & 15,05 \\
\hline Cantidad computadoras en hogar & 1 pcPCI & 13,45 \\
\hline Cantidad computadoras en hogar & Ninguna & 10,16 \\
\hline EduAdulAgrupado & Primario & 7,12 \\
\hline Internet en escuela & INTaveces & 7,10 \\
\hline ¿Con qué cosas de la lista asocias al PCI? & PCIFaceJuegos & 6,95 \\
\hline EstratosAgrupados & Estrato MedioAlto & 6,44 \\
\hline LaboralAdultoOK & Trabaja a veces & 6,23 \\
\hline LaboralAdultoOK & Desocupado & 6,18 \\
\hline EduAdulAgrupado & Primario Incompleto & 5,71 \\
\hline ¿Con qué cosas de la lista asocias al PCI? & PCIaudiovisual & 5,30 \\
\hline HabDocenteAgrupado & DocenteBueno & 4,64 \\
\hline Internet en escuela & INTsiempre & 4,02 \\
\hline Alumno trabaja o trabajó & AlumTrabaja & 3,89 \\
\hline HabAlumnoAgupado & AlumExcelente & 3,57 \\
\hline ¿Con qué cosas de la lista asocias al PCI? & PCIinternet & 2,87 \\
\hline
\end{tabular}




\begin{tabular}{|c|c|c|}
\hline ¿Con qué cosas de la lista asocias al PCI? & PCIescolar & 2,37 \\
\hline \multicolumn{3}{|l|}{ Tipo 3 (Casos: 905 Porcentaje: 28.43) } \\
\hline Etiqueta de la Variable & Categorías Caracteristicas & Test-value \\
\hline HabDocenteAgrupado & DocenteRegular & 21,16 \\
\hline Evaluar la netbook PCI & NETRegulares & 17,51 \\
\hline Cantidad computadoras en hogar & $+2 \mathrm{pcPCI}$ & 14,84 \\
\hline EstratosAgrupados & Estrato Alto & 14,62 \\
\hline ConsultaTICok & C Alumno & 14,33 \\
\hline ¿Con qué cosas de la lista asocias al PCI? & Con la netbook & 12,91 \\
\hline Internet en escuela & INTnunca & 11,05 \\
\hline LaboralAdultoOK & Trabaja & 9,46 \\
\hline Alumno trabaja o trabajó & AlumnNoTrabaja & 8,67 \\
\hline EduAdulAgrupado & Secundario & 6,05 \\
\hline HabAlumnoAgupado & AlumBueno & 5,46 \\
\hline EduAdulAgrupado & Superior & 4,81 \\
\hline Sexo & Femenino & 3,20 \\
\hline \multicolumn{3}{|l|}{ Tipo 4 (Casos: 393 Porcentaje: 12.35) } \\
\hline Etiqueta de la Variable & Categorías Caracteristicas & Test-value \\
\hline Evaluar la netbook PCI & NETns/nc & 16,30 \\
\hline HabDocenteAgrupado & DocenteMal & 10,37 \\
\hline Cantidad computadoras en hogar & Ninguna & 9,68 \\
\hline ¿Con qué cosas de la lista asocias al PCI? & PCIFaceJuegos & 8,69 \\
\hline ¿Con qué cosas de la lista asocias al PCI? & Con nada & 7,22 \\
\hline Alumno trabaja o trabajó & AlumTrabaja & 7,12 \\
\hline EduAdulAgrupado & Primario Incompleto & 6,83 \\
\hline LaboralAdultoOK & Desocupado & 5,72 \\
\hline ConsultaTICok & C Otros & 5,66 \\
\hline LaboralAdultoOK & Trabaja a veces & 5,49 \\
\hline HabAlumnoAgupado & AlumRegular & 5,16 \\
\hline Evaluar la netbook PCI & NETMalas & 4,58 \\
\hline ¿Con qué cosas de la lista asocias al PCI? & PCIotros & 4,03 \\
\hline Cantidad computadoras en hogar & $+2 \mathrm{pcNOPCI}$ & 3,54 \\
\hline EstratosAgrupados & Estrato MedioAlto & 3,10 \\
\hline HabAlumnoAgupado & AlumMal & 2,49 \\
\hline \multicolumn{3}{|l|}{ Tipo 5 (Casos: 393 Porcentaje: 12.35) } \\
\hline Etiqueta de la Variable & Categorías Caracteristicas & Test-value \\
\hline
\end{tabular}




\begin{tabular}{|l|c|c|}
\hline HabDocenteAgrupado & DocenteMal & 21,09 \\
\hline Evaluar la netbook PCI & NETMalas & 20,07 \\
\hline ConsultaTICok & C Otros & 15,95 \\
\hline Cantidad computadoras en hogar & +2 pcNOPCI & 15,58 \\
\hline HabAlumnoAgupado & AlumMal & 11,24 \\
\hline Internet en escuela & INTnunca & 10,76 \\
\hline ¿Con qué cosas de la lista asocias al PCI? & Con nada & 8,20 \\
\hline ¿Con qué cosas de la lista asocias al PCI? & PCIotros & 7,21 \\
\hline EduAdulAgrupado & Superior & 6,94 \\
\hline EstratosAgrupados & Estrato Alto & 6,76 \\
\hline Sexo & Masculino & 5,07 \\
\hline LaboralAdultoOK & Trabaja & 3,80 \\
\hline Evaluar la netbook PCI & NETRegulares & 3,47 \\
\hline Alumno trabaja o trabajó & AlumTrabaja & 2,67 \\
\hline HabAlumnoAgupado & AlumRegular & 2,67 \\
\hline
\end{tabular}

Fuente: elaboración propia 
Tabla IV

Composición de los dos ejes factoriales

\begin{tabular}{|c|c|c|c|}
\hline \multicolumn{4}{|l|}{ FACTOR 1} \\
\hline Variable & Categoría & Test-Value & Peso \\
\hline ConsultaTICok & C Docente & $-22,48$ & 1056,00 \\
\hline Evaluar la netbook PCI & NETMuy buenas & $-22,03$ & 573,00 \\
\hline HabDocenteAgrupado & DocenteExcelente & $-21,47$ & 359,00 \\
\hline HabDocenteAgrupado & DocenteBueno & $-19,74$ & 1121,00 \\
\hline Evaluar la netbook PCI & NETBuenas & $-15,69$ & 1135,00 \\
\hline Cantidad computadoras en hogar & $1 \mathrm{pcPCI}$ & $-15,51$ & 578,00 \\
\hline Internet en escuela & INTaveces & $-14,94$ & 956,00 \\
\hline Internet en escuela & INTsiempre & $-11,60$ & 500,00 \\
\hline EduAdulAgrupado & Primario & $-11,44$ & 1240,00 \\
\hline HabAlumnoAgupado & AlumExcelente & $-11,34$ & 1805,00 \\
\hline EstratosAgrupados & Estrato MedioAlto & $-10,71$ & 870,00 \\
\hline $\begin{array}{l}\text { ¿Con qué cosas de la lista asocias } \\
\text { al PCI? }\end{array}$ & PCIescolar & $-10,37$ & 879,00 \\
\hline \multicolumn{4}{|l|}{ MIDDLE AREA } \\
\hline $\begin{array}{l}\text { ¿Con qué cosas de la lista asocias } \\
\text { al PCI? }\end{array}$ & Con la netbook & 10,26 & 1353,00 \\
\hline EduAdulAgrupado & Superior & 11,34 & 626,00 \\
\hline ConsultaTICok & C Alumno & 11,74 & 1712,00 \\
\hline HabAlumnoAgupado & AlumMal & 13,79 & 79,00 \\
\hline HabDocenteAgrupado & DocenteRegular & 14,98 & 1228,00 \\
\hline Evaluar la netbook PCI & NETRegulares & 17,51 & 967,00 \\
\hline Cantidad computadoras en hogar & $+2 \mathrm{pcNOPCI}$ & 18,88 & 507,00 \\
\hline ConsultaTICok & C Otros & 19,22 & 271,00 \\
\hline EstratosAgrupados & Estrato Alto & 19,36 & 636,00 \\
\hline Internet en escuela & INTnunca & 22,21 & 1727,00 \\
\hline Evaluar la netbook PCI & NETMalas & 23,28 & 337,00 \\
\hline HabDocenteAgrupado & DocenteMal & 25,05 & 475,00 \\
\hline \multicolumn{4}{|l|}{ FACTOR 2} \\
\hline Variable & Categoría & Test-Value & Peso \\
\hline Cantidad computadoras en hogar & $+2 \mathrm{pcPCI}$ & $-26,86$ & 1268,00 \\
\hline HabDocenteAgrupado & DocenteRegular & $-17,54$ & 1228,00 \\
\hline LaboralAdultoOK & Trabaja & $-16,50$ & 2762,00 \\
\hline
\end{tabular}




\begin{tabular}{|l|c|c|l|}
\hline Alumno trabaja o trabajó & AlumnNoTrabaja & $-14,14$ & 1891,00 \\
\hline Evaluar la netbook PCI & NETBuenas & $-14,02$ & 1135,00 \\
\hline $\begin{array}{l}\text { ¿Con qué cosas de la lista asocias } \\
\text { al PCI? }\end{array}$ & Con la netbook que t & $-13,49$ & 1353,00 \\
\hline Evaluar la netbook PCI & NETRegulares & $-12,70$ & 967,00 \\
\hline ConsultaTICok & C Alumno & $-12,00$ & 1712,00 \\
\hline EduAdulAgrupado & Secundario & $-11,99$ & 1063,00 \\
\hline EstratosAgrupados & Estrato Alto & $-11,55$ & 636,00 \\
\hline $\begin{array}{l}\text { ¿Con qué cosas de la lista asocias } \\
\text { al PCI? }\end{array}$ & PCIescolar & $-9,41$ & 879,00 \\
\hline HabDocenteAgrupado & DocenteBueno & $-6,58$ & 1121,00 \\
\hline M I D D L E A R E A & Trabaja a veces & 11,23 & 191,00 \\
\hline LaboralAdultoOK & AlumMal & 11,45 & 79,00 \\
\hline HabAlumnoAgupado & NETMalas & 13,75 & 337,00 \\
\hline Evaluar la netbook PCI & Primario Incompleto & 14,00 & 200,00 \\
\hline EduAdulAgrupado & AlumTrabaja & 14,09 & 1288,00 \\
\hline Alumno trabaja o trabajó & C Otros & 14,56 & 271,00 \\
\hline ConsultaTICok & Con nada & 15,26 & 150,00 \\
\hline $\begin{array}{l}\text { ¿Con qué cosas de la lista asocias } \\
\text { al PCI? }\end{array}$ & DocenteExcelente & 15,40 & 359,00 \\
\hline HabDocenteAgrupado & PCIFaceJuegos & 16,62 & 216,00 \\
\hline $\begin{array}{l}\text { ¿Con qué cosas de la lista asocias } \\
\text { al PCI? }\end{array}$ & DocenteMal & 19,10 & 475,00 \\
\hline HabDocenteAgrupado & NETns/nc & 19,28 & 171,00 \\
\hline Evaluar la netbook PCI & 19,51 & 329,00 \\
\hline Cantidad computadoras en hogar & \multicolumn{2}{|l}{} \\
\hline
\end{tabular}

Fuente: elaboración propia 